\title{
Power analysis for multivariate and repeated measures designs: A flexible approach using the SPSS MANOVA procedure
}

\author{
ELIZABETH J. D'AMICO \\ University of California, San Diego, La Jolla, California \\ TORSTEN B. NEILANDS \\ University of Texas, Austin, Texas \\ and \\ ROBERT ZAMBARANO \\ PPD Informatics, Austin, Texas
}

\begin{abstract}
Although power analysis is an important component in the planning and implementation of research designs, it is often ignored. Computer programs for performing power analysis are available, but most have limitations, particularly for complex multivariate designs. An SPSS procedure is presented that can be used for calculating power for univariate, multivariate, and repeated measures models with and without time-varying and time-constant covariates. Three examples provide a framework for calculating power via this method: an ANCOVA, a MANOVA, and a repeated measures ANOVA with two or more groups. The benefits and limitations of this procedure are discussed.
\end{abstract}

Power analysis is often overlooked in the design of scientific studies because of the belief that the calculations and information required are not easy to obtain. As a result, two common mistakes may occur: the selection of an insufficient sample size (Muller, LaVange, LandesmanRamey, \& Ramey, 1992), or the inclusion of too many participants (Bird \& Hall, 1986; Muller et al., 1992). Readers are most likely familiar with how these design mistakes may lead to the two types of error associated with statistical tests. Type I error occurs when the null hypothesis is falsely rejected. Thus, a significant difference is indicated when none exists. The likelihood of making a Type I error that a researcher is willing to tolerate is $\alpha$ ( $\alpha$ is typically set at .05). By contrast, a Type II error occurs when the null hypothesis fails to be rejected by the investigator when in fact that null hypothesis is false in the population. In this case, no significant difference is statistically indicated from the sample data when a difference does exist in the population. The likelihood of making a Type II error is $\beta$.

Power, or $1-\beta$, is the probability of correctly rejecting the null hypothesis when it is false in the population(Cohen,

The authors thank Mark Appelbaum and Jesse Canchola for their comments on a previous draft of this manuscript. The authors also thank reviewers Brian O’Connor, George A. Morgan, Nancy L. Leech, and an anonymous reviewer for their helpful suggestions on an earlier version of this manuscript. T. Neilands is now affiliated with the Center for AIDS Prevention Studies, University of California, San Francisco. Correspondence concerning this article should be addressed to E. J. D'Amico, University of California, San Diego, 9500 Gilman Dr. (0109), La Jolla, CA 92093-0109(e-mail: edamico@psy.ucsd.edu).
1988). A priori power analysis enables researchers to design better studies and determine whether the effort, time, and money required by their research designs are warranted. Although research designs have become more complicated in recent years (e.g., multivariate and repeated measures models), corresponding power analyses to evaluate the complexity and feasibility of these designs are typically not performed (Muller et al., 1992).

This may be due, in part, to the fact that articles and books that address power analysis for more complex designs such as general linear models (GLMs) have not been available until recently, particularly for repeated measures models (Stevens, 1996). However, recent articles have begun to focus on power for ANOVA designs with one repeated measure (e.g., split-plot, ANCOVA, correlated samples; Levin, 1997) and for two-factor repeated measures ANOVA (Potvin \& Schutz, 2000).

An accessible and general purpose approach to power analysis for complex designs is long overdue. It is common, for example, for researchers to investigate several different design possibilities for any given research proposal (e.g., MANOVA, ANCOVA, repeated measures); using a hand calculator and sets of power tables to determine power for each design would be an onerous approach. Fortunately, statistical software packages are available to perform power calculations. For example, Muller and his colleagues (Muller et al., 1992) discuss power for GLMs in their review paper and include an SAS program (SAS, 1990) that computes power for these models. Use of this program, however, requires strong knowledge of the SAS programming language because the computations are 
complex; many users may not have this level of expertise. In addition, such programs may generate power calculations for a select subset of available models of interest; locating and using different programs to compute power for different models can be inconvenient and time consuming.

Software packages are available, however, that are "user-friendly" and allow the researcher more flexibility in evaluating the research design. For instance, the SPSS, Inc. product called Sample Power (Borenstein, Rothstein, \& Cohen, 1997) fits regression, ANOVA, and ANCOVA models, and also offers power calculations for mean comparisons and tests of proportions. In addition, the NCSS Power Analysis and Sample Size program (PASS) offers power calculations for linear and nonlinear models, as well as asymptotic and some exact tests of equality of proportions (Hintze, 2000). Power analysis programs are also available on the Internet (Lenth, 2000a). Conspicuously absent from these programs, however, are power computations for multivariate general linear models that feature multiple dependent variables.

By contrast, the present paper discusses a method that comes as part of the general SPSS statistical software package and covers most GLM designs. Thus, with this method, it is possible to examine models with multiple dependent variables, as well as single-dependent variable models. Limited knowledge of SPSS programming language is required if the version being used includes the SPSS graphical user interface (Version 6 and above).

The present paper provides three examples of common research designs and the corresponding power analysis for each of these designs. These examples were chosen because they illustrate the flexibility of the SPSS method and permit one to examine less commonly documented designs in the power analysis literature. Using the MATRIX DATA and MANOVA procedures (SPSS, 1997a, 1999), power is calculated for an analysis of covariance (ANCOVA) design, a multivariate analysis of variance (MANOVA) design, and a repeated measures design wherein the data include both between- and within-subjects factors.

\section{STATISTICAL EXAMPLES CALCULATING POWER}

\section{Overview}

The SPSS MANOVA procedure is quite flexible and can be used to fit the following models: one-way ANOVA, factorial ANOVA, ANCOVA, MANOVA, MANCOVA, and repeated measures models with and without timevarying and time-constant covariates. Researchers may also use the MANOVA procedure to fit linear regression models. Readers should note that historically SPSS used MANOVA in the pulldown menu to perform general linear modeling, but in Versions 7.0 and above, the pulldown menu analysis is changed to GLM. Thus, MANOVA must now be accessed by typing syntax.

The SPSS syntax used to fit the models is typically straightforward. In a few rare instances (e.g., repeated measures models with time-constant covariates), it may be more difficult. Interested readers may want to review the SPSS Advanced Statistics Guide (SPSS, 1997a, 1999) for more information on the MANOVA procedure and syntax. Overall, if the applied researcher has both the SPSS Base and Advanced Statistics (renamed SPSS Advanced Models in Version 9.0 and higher) modules, power computation is available for the most simple and the most complicated research designs.

When researchers calculate power prior to data collection, two similar questions are typically asked: (1) What is the power of the hypothesis test to detect an effect as a function of the significance criterion, effect size, and the sample size? and (2) what should the sample size be as a function of effect size, significance criterion, and power?

Before power can be calculated with the following procedure, some important information must be gathered. Access to the relevant literature (e.g., past published research or available pilot data) is crucial, because the following data are needed: (1) expected means and standard deviations for the dependent variables of interest for all assessment periods, (2) the number of subjects proposed for the current study of interest, and (3) correlations between the dependent variables (if these are not available, a range of correlations, .10 to .90 , may be examined). An examination of the literature should provide researchers with access to studies that have similar designs, thus providing the necessary data. Alternatively, a strong theory can enable a researcher to make useful guesses for values that would provide theoretical support.

\section{Effect Size}

When using the SPSS method described in this paper, one does not need to calculate effect size measures because the technique is based on the sample statistics actually being modeled and tested. It is often the case, however, that researchers may be more comfortable working with effect sizes, particularly if no expected means, standard deviations, or intervariable correlations are available from pilot data or previously published research. A familiar effect size to many researchers is Cohen's $d$, which is defined differently for various experimental designs (Cohen, 1988). For example, in a two-group design with a single outcome variable (i.e., the independent samples $t$ test), $d$ is equal to the difference of the means for the groups divided by the pooled standard deviation (Cohen, 1988). Based on Cohen's $d$, effect sizes can be small (.30), medium (.50), or large (.70).

As part of its output, SPSS produces an index of effect size, partial $\eta^{2}$, from the values that are supplied. In the context of general linear models, the partial $\eta^{2}$ represents the proportion of variability in the dependent variable(s) that is explained by the independent variable(s) (SPSS, 1997a). The following are small, medium, and large effects for $\eta^{2}$, respectively: .01, .06, and .14 (Stevens, 1996). Although it can be advantageous to conduct a power analysis on the basis of expected effect sizes alone, important 
Table 1

Means and Standard Deviations $(S D)$

for Patient Anxiety Ratings, Number of Siblings, and Parent Anxiety Level by Age Group

\begin{tabular}{|c|c|c|c|c|c|c|}
\hline & \multicolumn{2}{|c|}{$\begin{array}{l}\text { Patient } \\
\text { Anxiety }\end{array}$} & \multicolumn{2}{|c|}{$\begin{array}{c}\text { Number of } \\
\text { Siblings } \\
\end{array}$} & \multicolumn{2}{|c|}{$\begin{array}{c}\text { Parent } \\
\text { Anxiety }\end{array}$} \\
\hline & $M$ & $S D$ & $M$ & $\overline{S D}$ & $\bar{M}$ & $S D$ \\
\hline $\begin{array}{l}\text { Children } \\
\quad \text { (age 6-12) }\end{array}$ & 7.5 & 1.9 & 3 & 1 & 4 & 2.3 \\
\hline $\begin{array}{l}\text { Adolescents } \\
\quad \text { (age 13-19) } \\
\text { Adults }\end{array}$ & 6.8 & 2.5 & 2 & 2 & 5 & 1.4 \\
\hline $\begin{array}{l}\text { Adults } \\
\quad \text { (age 20-45) }\end{array}$ & 7.1 & 2.1 & 4 & 1 & 6 & 1.8 \\
\hline
\end{tabular}

Note- Participants' anxiety ratings could range from 1 (not at all anxious) to 10 (very anxious).

issues may be ignored that are addressed by using the present procedure, such as a more detailed analysis of both the research design and specific hypotheses (Lenth, 2000b).

\section{ANCOVA Design}

Table 1 provides data for the first example. In this case, the researcher is interested in whether age affects level of reported anxiety. Power will be determined for the following research question: Is a patient's anxiety level (pat_anx) associated with his/her age (agegroup), controlling for number of siblings (sibnumbr) and parent level of anxiety (prnt_anx)? To calculate power for a study, researchers would similarly provide means and standard deviations from pilot data, previous literature, or guesses informed by theory. The following SPSS syntax ${ }^{1}$ is used to generate a matrix of sufficient statistics (i.e., means, standard deviations, cell $n \mathrm{~s}$, and intervariable correlations) from the data provided in Table 1 and compute power using the MANOVA command in SPSS Windows format. ${ }^{2}$ Note the spaces after rowtype_and before the sd and corr lines:

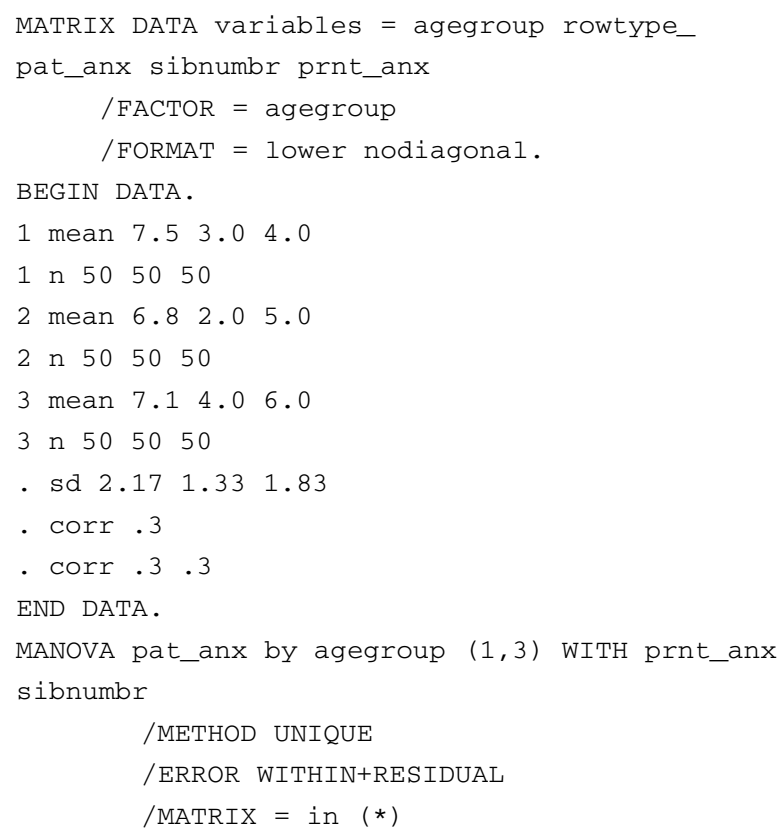

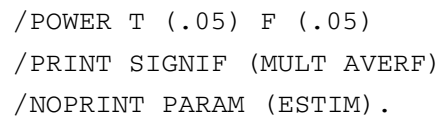

The means for each group (e.g., 1 mean 7.5 3.0 4.0 are the data for Group 1), number of participants (e.g., 1 n 50 5050 are the data for Group 1), standard deviations (i.e., . sd 2.171 .331 .83 ), and correlations (i.e., . corr .3 and . corr .3 .3) are entered directly into the syntax file using a "begin data" statement following the MATRIX DATA command. A matrix data file containing the sufficient statistics for the analysis is then generated with these original input data; thus, the newly created SPSS sufficient statistics data file will contain means, standard deviations, and correlations for the following variables: pat_anx, sibnumbr, and prnt_anx. The MANOVA command will use this generated matrix data file to calculate power. In this example, a sample of 50 has been chosen for each group, with a total number of 150 participants for this example. Note that a mean standard deviation is entered in the syntax file, which is based on data from the three groups in Table 1. For example, the mean standard deviation for patient anxiety level is $(1.9+2.5+2.1) / 3=2.17$. For this example, a correlation of .30 between all of the variables was chosen; correlations can be changed, however, if a review of previous data indicates higher or lower correlations.

Figure 1 provides the output and the observed power for the group main effect after the MANOVA syntax has been run. Recall that the question of interest was whether a patient's anxiety level would differ depending upon age. Cohen (1988) has suggested that .80 or higher is an adequate level of power. Thus, results from this power analysis indicate that more participants may be needed in order to obtain a satisfactory degree of power, since power for the group main effect is .74. On the basis of this analysis, the investigators could conclude that 150 participants, with 50 participants in each group, is not an adequate sample size for obtaining the significant effect of interest. They might want to include a few more participants in each group in order to obtain a more satisfactory power level.

To examine what would be required in order to obtain a power of .80 , or if the obtained power was not satisfactory after this analysis had been conducted, it is possible to go back to the original syntax file and change some of the matrix data. For example, power could be calculated for a larger sample (e.g., $N=200$ ). Remember, the number of participants in each group must be changed in the syntax file. For example, if a total $N$ of 200 was chosen, an $N$ of 66 could be input for Group 1 (i.e., 1 n 6666 66), Group 2, $N=67$, and Group $3, N=67$. The same syntax would be run again and a new power calculation would be output. For this example, power of .86 would be obtained.

Power may also be recalculated by keeping the same sample size and changing the correlation value. For example, perhaps previous literature has reported correlations ranging from .30 to .60 . Power values could be calculated for the same sample size using correlations of $.30, .40, .50$, and .60 to determine whether the power value was suffi- 


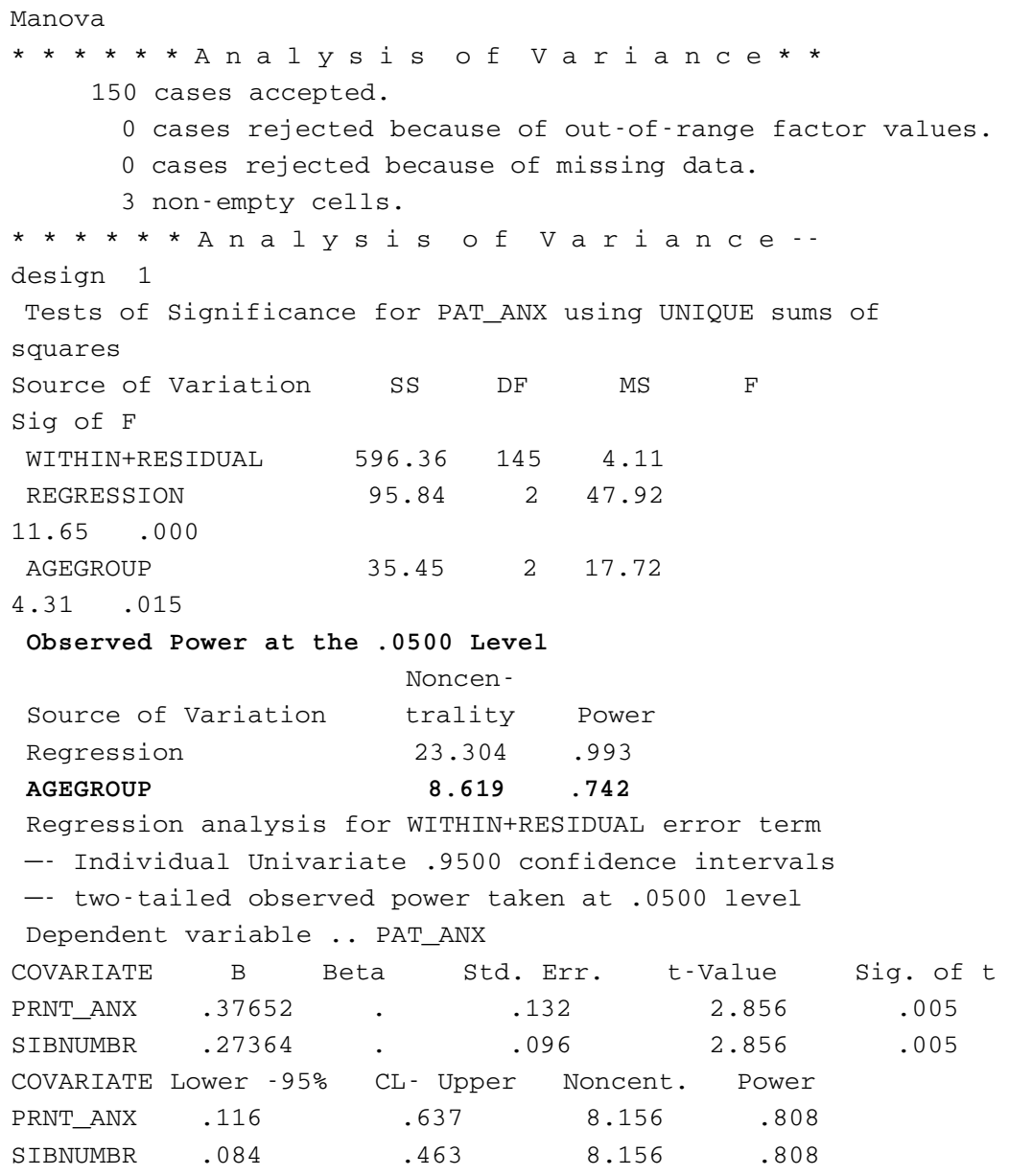

Figure 1. SPSS output for the ANCOVA procedure with observations of power.

cient at a prespecified correlation level. It is always safer, however, to increase sample size to ensure adequate power rather than to depend on the strength of a correlation.

\section{MANOVA Design}

In this example, the research question is whether differences exist among three ethnic groups (Caucasians, Hispanics, and Asian Americans) on ratings of the risk and benefit of alcohol consumption (total $N=60$ ). The syntax is the following:

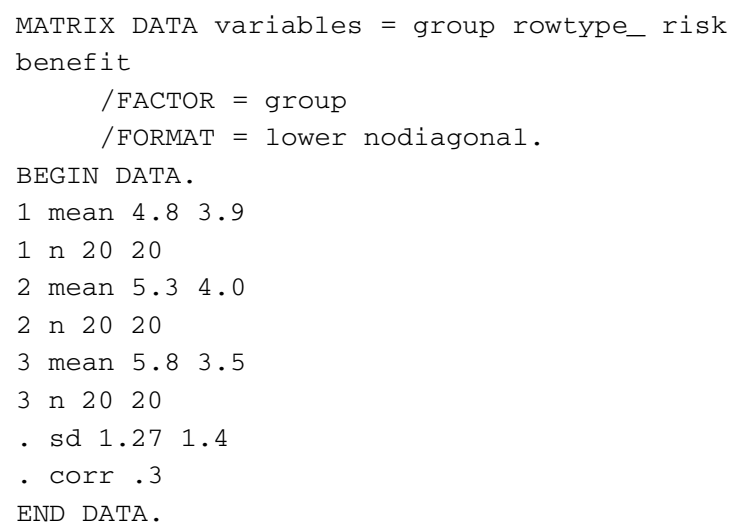

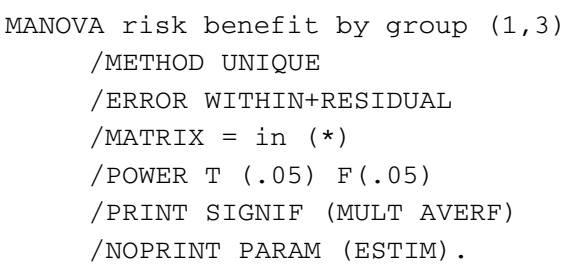

The correlations are again specified to be .30 between the variables. Power $=.57$ for the univariate test statistic for risk, and power $=.17$ for the benefit variable. Power for the multivariate test statistic of the group effect on the pooled dependent variable combination was .65. Therefore, more participants are needed to obtain adequate power for the current research design. By changing the total number of participants in the syntax file to 90 and adding 10 participants to each group $(N=30)$, one obtains a more acceptable level of power for risk (power $=.77$ ); however, the power for benefit is still quite low (power = .23). By contrast, the power for the multivariate test statistic is more than adequate (.85). If the researcher is more interested in the ethnic difference for benefit ratings, more participants are needed in order to test this hypothesis adequately. In contrast, power is sufficient for the hypothesis concerning risk ratings. 


\section{Repeated Measures Design}

In this example, three groups (expgroup) are measured at three different time points. Two of these groups receive an intervention and one is a control group. The means in the syntax file below are the groups' self-reported ratings of depression over three time points. The test of the depression effect across time is defined as "depress" in the syntax shown below. The interventions took place after the second assessment. The research question in this example is whether a behavioral intervention or a cognitive intervention will be effective in decreasing participants' self-reported depression. The interventions are compared to a wait-list control group. The following syntax is run:

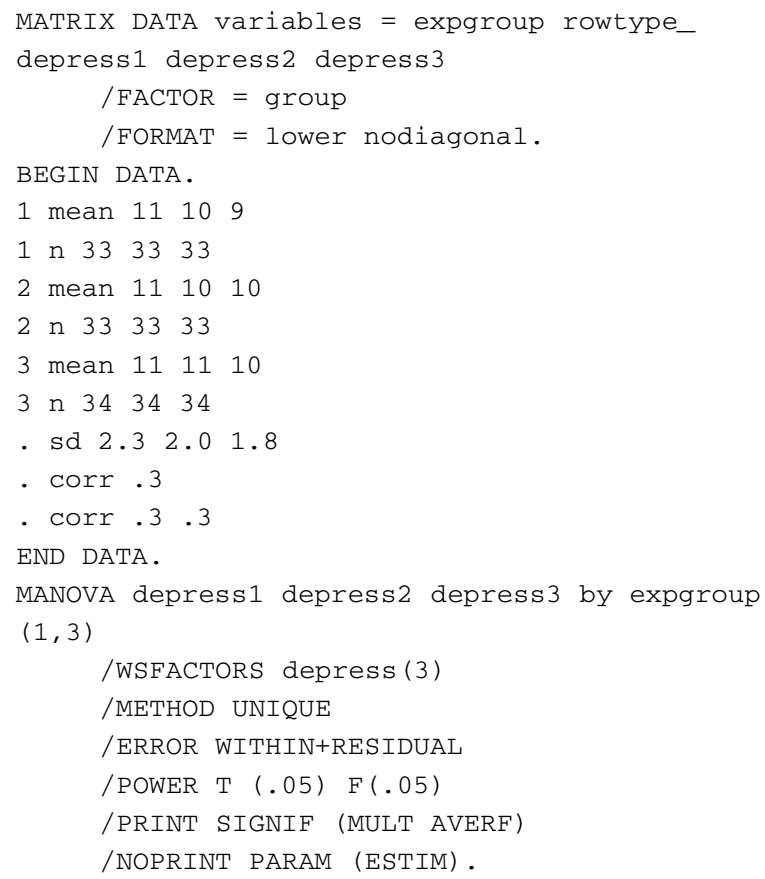

As for the two previous examples, correlations are specified to be .30 between the variables. In this example, 100 total participants are specified and the obtained power is .57 for the multivariate group $\times$ measurement occasion interaction. The results indicate that this power level is not sufficient. If one changes the number of participants to 200 in this example (approximately 66 per group), power $=.89$ for the group $\times$ measurement occasion interaction. Thus, the results of this power analysis indicate that a satisfactory degree of power has been obtained through increasing the sample size. Note that this example illustrates the flexibility of the SPSS MANOVA power analysis for handling designs with unbalanced data. Researchers may want to test the impact of different group sizes on the resulting power values.

\section{CONCLUSIONS AND LIMITATIONS}

Power analysis can clarify hypotheses and determine whether there is a sufficient chance of rejecting the null hypothesis when it is false. It is an objective way to eval- uate a research design and minimize Type II errors. In order to use the procedure, researchers must have access to a statistical software package such as SPSS that produces power computations as part of its general linear model procedure output and accepts sufficient statistics as input data. Researchers who plan to use SPSS for these analyses need the SPSS Base and Advanced Statistics modules. In addition, a basic set of parameter estimates of the data is needed (e.g., means, standard deviations, and correlations), although these may be difficult to generate because such data may not always be available. As a second choice, researchers may want to collect pilot data to get rough estimates of the statistics of interest. If this is not possible, the only recourse is to generate a range of likely means, standard deviations, and correlations on the basis of theory.

Note that researchers must also have an understanding of effect size, because appropriate mean differences must be determined for this analysis, and the procedure requires the conversion of effect sizes into expected mean differences. Although this is a limitation, it could also be considered a strength, because it requires that investigators be familiar with data, measures, and design considerations in their area of study (Lenth, 2000b).

This method may also be tedious for the researcher who is interested in computing power for several different independent and dependent variables, because the means, standard deviations, and correlations must be input for each separate analysis. The procedure must thus be run multiple times; but the syntax can be placed in one file and run at the same time once the researcher has determined the variables of interest. Once the syntax file is saved, different $N_{\mathrm{s}}$, means, and standard deviations can simply be adjusted for each independent analysis if power is not sufficient and must be recalculated.

Finally, the SPSS MANOVA procedure is limited in that it cannot be used to examine power for designs with categorical outcomes (e.g., logistic regression, loglinear models), simultaneous equations (e.g., structural equation modeling), or mixed effects models or general linear models with heterogeneous covariance structures (Littell, Milliken, Stroup, \& Wolfinger, 1996). Interested readers may use the NCCS PASS program for logistic regression models (Hintze, 2000), or Egret SIZ for logistic regression and Cox proportional hazards models (Cytel Software, 1998). Muthén and Curran (1997), as well as Satorra and Saris (1985), provide examples of power computation methods for latent variable models.

As with all power computations, lower levels of power may be found for some effects (e.g., interactions) and higher power may be found for other effects (e.g., main effects). Although this may be considered a dilemma, the present procedure can help inform the researcher and contribute to a reformulation of hypotheses before the study begins. For example, results may indicate that more participants are needed for one to adequately address a hypothesis related to an interaction. Thus, although power may be sufficient for main effects, if the interaction ad- 
dresses the research question of interest, then the researcher has learned that the sample size must be increased if he/she wants to test this hypothesis adequately. ${ }^{3}$ The disparity in power for the different effects may also suggest that the proposed design is not the best or most efficient way to test the particular hypothesis.

In summary, the present procedure offers a flexible approach that allows a rapid examination of power for a wide range of designs before data collection begins. Hypotheses for a variety of multivariate general linear models, many of which are not accessible in other power calculation software programs or tables found in statistical textbooks, can therefore be evaluated so that the underlying research questions can be adequately assessed.

\section{REFERENCES}

BIRD, K. D., \& HALl, W. (1986). Statistical power in psychiatric research. Australian \& New Zealand Journal of Psychiatry, 20, 189 200.

Borenstein, M., Rothstein, H., \& Cohen, J. (1997). Sample power: Release 1.00. Chicago, IL: SPSS, Inc.

COHEN, J. (1988). Statistical power analysis for the behavioral sciences (rev. ed.). New York: Academic Press.

Cytel Software (1998). Egret SIZ: An advanced tool for power and sample size estimation. [On line]. http://www.cytel.aa.psiweb.com/ products/egret/egret3.html.

HinTZE, J. (2000). NCSS PASS. [On line]. http://www.ncss.com/pass.html.

LENTH, R. V. (2000a). JAVA applets for power and sample size. [On line]. http://www.stat.uiowa.edu/ rlenth/Power/.

LENTH, R. V. (2000b). Two sample-size practices that I don't recommend. Paper presented at the Joint Statistical Meetings of the American Statistical Association, Indianapolis.

LEVIN, J. R. (1997). Overcoming feelings of powerlessness in "aging" researchers: A primer on statistical power in analysis of variance designs. Psychology \& Aging, 12, 84-106.

Littell, R. C., Milliken, G. A., Stroup, W. W., \& Wolfinger, R. D. (1996). SAS system for mixed models. Cary, NC: SAS Institute, Inc. Muller, K. E., LaVAnge, L. M., Landesman-Ramey, S., \& Ramey, C. T. (1992). Power calculations for general linear multivariate models including repeated measures applications. Journal of the American Statistical Association, 87, 1209-1226.
Muthén, B., \& Curran, P. (1997). General longitudinal modeling of individual differences in experimental designs: A latent variable framework for analysis and power estimation. Psychological Methods, 2, 371-402.

Potvin, P. J., \& Schutz, R. W. (2000). Statistical power for the twofactor repeated measures ANOVA. Behavior Research Methods, Instruments, \& Computers, 32, 347-356.

SAS Institute, InC. (1990). SAS/STAT user's guide (Vol. 1). Cary, NC: Author.

Satorra, A., \& SARIS, W. (1985). Power of the likelihood ratio test in covariance structure analysis. Psychometrika, 51, 83-90.

SPSS, INC. (1997a). SPSS advanced statistics 7.5. Chicago, IL: Author. SPSS, INC. (1997b). SPSS 7.5 statistical algorithms. Chicago, IL: Author. SPSS, INC. (1999). SPSS 10.0 syntax reference guide. Chicago, IL: Author. STEVENS, J. (1996). Applied multivariate statistics for the social sciences. Mahwah, NJ: Erlbaum.

\section{NOTES}

1. Some readers may be more familiar with the point and click pulldown menus that allow one to run different analyses in SPSS. As noted earlier, in Versions 7.0 and above, the pulldown menu no longer includes the MANOVA command and instead includes a GLM command. The present procedure therefore requires the user to open a new syntax window in SPSS and type in the syntax provided.

2. It is possible to perform this analysis using SPSS Version 6.1 or higher on several different platforms, such as Windows 95, 98, NT, and 2000, as well as the Mac OS, UNIX, and VMS. The SPSS Statistical Algorithms Guide (SPSS, 1997b) contains all the formulas used for power calculations in this procedure. These formulas may also be found on line at the SPSS FTP site: ftp://ftp.spss.com/pub/spss/statistics/spss/algorithms/.

3. Granting agencies often ask for information regarding power. The following is one example of how to write up this information:

Power was calculated using the SPSS procedure, which requires the number of participants for each group, estimated means and standard deviations for the dependent variables, and estimated correlations between the variables. Means and standard deviations were taken from [insert reference or pilot data]. Correlations were not available from this reference, so power was calculated using a conservative correlation of .10. Even when this small correlation was used, power for detecting the group $\times$ time interaction was adequate (e.g., .78).

(Manuscript received August 29, 2000; revision accepted for publication July 13, 2001.) 